\title{
Phenotypic consequences in black South African Fanconi anemia patients homozygous for a founder mutation
}

\author{
Candice Feben, MBBch, FCMG (SA)'1, Jennifer Kromberg, MA, PhD², \\ Rosalind Wainwright, MBBch, FCPaed (SA) ${ }^{3}$, David Stones, MBBch, FCPaed (SA)4, \\ Chris Sutton, MBBch, FCPaed (SA) ${ }^{5}$, Janet Poole, MBBch, FCPaed (SA) ${ }^{6}$, \\ Tabitha Haw, MSc med (Genetic counselling) ${ }^{1}$ and Amanda Krause, MBBch, PhD ${ }^{1}$
}

Purpose: Fanconi anemia is a genotypically and phenotypically heterogeneous condition, characterized microscopically by chromosomal instability and breakage. Affected individuals manifest growth restriction and congenital physical abnormalities; most progress to hematological disease including bone marrow aplasia. Black South African Fanconi anemia patients share a common causative founder mutation in the Fanconi G gene in 80\% of cases (637_643delTACCGCC). The aim of this study was to investigate the genotype-physical phenotype correlation in a cohort of individuals homozygous for this mutation.

Methods: Thirty-five black patients were recruited from tertiary level hematology/oncology clinics in South Africa. Participants were subjected to a comprehensive clinical examination, documenting growth, congenital anomalies, and phenotypic variability.
Results: Descriptive statistical analysis showed significant growth abnormalities in many patients and a high frequency (97\%) of skin pigmentary anomalies. Subtle anomalies of the eyes, ears, and hands occurred frequently $(\geq 70 \%)$. Apart from malformations of the kidney (in 37\%) and gastrointestinal tract (in 8.5\%), congenital anomalies of other systems including the cardiovascular and central nervous systems, genitalia, and vertebrae were infrequent $(<5 \%)$.

Conclusion: The diagnosis of Fanconi anemia in black South African patients before the onset of hematological symptoms remains a clinical challenge, with the physical phenotype unlikely to be recognized by those without dysmorphology expertise.

Genet Med advance online publication 26 December 2013

Key Words: black South African patients; FANCG founder mutation; Fanconi anemia; genotype-physical phenotype correlation

\section{INTRODUCTION}

Fanconi anemia (FA) is a rare condition of chromosomal instability, inherited in either an autosomal-recessive or an $\mathrm{X}$-linked manner. ${ }^{1,2}$ It is both genotypically and phenotypically heterogeneous and is a disorder that affects individuals of diverse ethnic origin.

Clinically, FA is characterized by a broad spectrum of congenital anomalies, principally involving growth, skin pigmentation, and morphogenesis. A heterogeneous spectrum of abnormalities of the skeletal, cardiovascular, genitourinary, gastrointestinal, and central nervous systems is well documented, ${ }^{3}$ as is endocrine dysregulation, involving glucose homeostasis, growth hormone, and thyroid function. ${ }^{4}$ Bone marrow failure remains, however, the hallmark of the disease, with initial pancytopenia and progression to aplastic anemia, acute myeloid leukemia, or myelodysplastic syndrome. ${ }^{5}$ A predisposition to solid tumors of the head, neck, liver, esophagus, and female genital tract is also well described and has become more prevalent in developed countries, as the treatment for the hematological complications of the condition has improved. Bone marrow transplantation now provides the possibility of cure for the hematological complications of the condition. ${ }^{6}$

Fifteen complementation groups of FA have been described, each associated with numerous causative mutations within specific genes. The genes have been named FANC A, B, C, D1, $D 2, E, F, G, I, J, L, M, N, O$, and $P .^{2}$ The gene products are all thought to function in a complex S-phase-specific cellular response to damaged DNA. ${ }^{7}$ Worldwide, as documented in the International Fanconi Anemia Registry, FANC A, $C$, and $G$ mutations are the most prevalent, accounting for 60,16 , and $10 \%$ of cases, respectively. ${ }^{8}$

In South Africa, black individuals with FA share a common causative founder mutation (637_643delTACCGCC) in the FANCG gene with $80 \%$ of cases being homozygous for this mutation. Haplotype analysis of the FANCG gene is consistent with a single origin for this mutation, predating the arrival of Bantu speakers in Southern Africa, in 400 AD. On the basis of the mutation frequency found in this population group, the incidence of FA is predicted to approximate $1 / 40,000 .{ }^{9}$ Notwithstanding advances in standards of care, the health-care 
system in South Africa remains underdeveloped and is largely based on semi-urban and rural primary health-care clinics that are predominantly staffed by nurses. Despite the severity of FA, it has previously been postulated that only a small percentage of affected patients are recognized in these local and regional health-care institutions and referred for tertiary management ${ }^{9,10}$ although the reasons for this remain unclear.

Before this study, no comprehensive genotype-phenotype correlation study had been performed in the subgroup of black South African patients homozygous for the founder FANCG mutation. This patient group represents a unique cohort for a mutation-specific genotype-phenotype correlation study as all the patients are homozygous for the same FA causing mutation and are thus truly genetically homogeneous at this locus.

The aim of this study was to investigate whether a specific physical phenotype exists for individuals homozygous for the founder mutation and whether this phenotype differs significantly from that described in other FA cohorts. We also assessed whether the physical phenotype could be used to identify black children with FA at primary health-care facilities at a stage before bone marrow failure developed so as to facilitate more expedient referral to tertiary institutions.

\section{MATERIALS AND METHODS}

The study was conducted at tertiary level hematology/oncology clinics in the cities of Johannesburg, Bloemfontein, and Polokwane in three provinces of South Africa, between October 2009 and July 2011. Black patients, confirmed homozygous for the FANCG founder mutation by direct polymerase chain reaction analysis, were recruited to participate in the study. Although it is recognized that chromosome breakage testing remains the gold standard for diagnosing FA internationally, in South Africa, molecular techniques are used more commonly to confirm the diagnosis. As the founder mutations have been identified in certain population groups, targeted molecular testing is not only technically simpler but also more cost effective in a resourcerestricted setting. Complementation analysis was not performed on these individuals to confirm that the mutation is the sole source of the FA as this testing is not available diagnostically in South Africa. However, Morgan et al., ${ }^{9}$ in elucidating the founder mutation, performed a complementation analysis in their cohort and established that the FANCG protein was undetectable in all cell lines tested. The deletion mutation produces a truncated protein of 217 amino acid residues and is thought to be pathogenic. ${ }^{9}$

Altogether, 35 unrelated black patients, homozygous for the founder mutation on molecular testing, were included. In cases of affected sibling pairs, only the elder sibling was chosen to participate to reduce the possible influence of familial shared physical characteristics.

Participation included a comprehensive clinical examination and a concurrent review of the patient's hospital records. The clinical examination aimed to document phenotypic variation including growth with measurements of current weight, height, and head circumference; facial variation involving the eyes and ears, with measurements of the palpebral fissure and pinna length, respectively; and upper and lower limb variation, with particular focus on anomalies of the radial ray and hands and skin pigmentary abnormalities. All measurements were based on the techniques described in "Handbook of Physical Measurements"11 and were plotted on relevant growth charts for comparison with the age-related mean. Although it is recognized that these growth and physical parameter charts were derived from predominantly Caucasian population groups, no similar charts have been developed for use in black South African patients to date.

Major malformations including renal, urogenital, cardiovascular, skeletal, and central nervous system anomalies were documented, if noted, on the clinical examination, and confirmed with further investigations including X-ray and ultrasound imaging. Renal ultrasound evaluations had been performed in $89 \%(31 / 35)$ of the patient cohort. Information from the patients' hospital records was used to document the age of presentation to the hematology/oncology clinic and the clinical symptoms at initial presentation.

Although it is recognized that endocrine anomalies are well described in FA cohorts, it was not possible to perform oral glucose tolerance testing and growth hormone stimulation testing on all patients owing to cost constraints. Samples taken for random glucose and thyroid function tests were subject to storage and transportation problems and could not be analyzed.

Descriptive statistical analysis of the data was performed. The median current age and median age of presentation with symptoms in keeping with FA was calculated. Growth measurements were converted to $z$-scores utilizing World Health Organization Anthro and World Health Organization Anthroplus software. Weight for age $z$-scores could only be calculated in children younger than the age of 10 years as the World Health Organization calculators do not allow for a calculation in those older than 10 years of age. The $z$-scores were used to compare the growth measurements of the study group with a cohort of healthy (cohort of rural South African children presumed not to have significant chronic illness or disease) black South African children as published by Kimani-Murage et al. ${ }^{12}$ The frequency of individuals with weight for age and height for age $z$-scores $<-2$ in the two groups was compared using Fisher's exact test. The frequency of each described physical phenotypic parameter was calculated. The frequency of certain physical characteristics in the black South African cohort was compared with frequency figures previously reported in other FA cohorts using Fisher's exact test. Calculated $P$ values were deemed significant if $<0.05$.

The study was approved by the Research Ethics Committees of the University of the Witwatersrand, The University of the Free State, and The University of Limpopo.

\section{RESULTS}

The median age of the 35 patients attending the hematology/ oncology clinics at the time of the study was 9 years, 11 months (range: 3 years to 17 years, 5 months). The most frequent initial presenting complaint was recurrent epistaxis. None of the 35 patients presented or were referred to tertiary hematology/oncology clinics on the basis of identified congenital 
malformations or poor growth. The reasons for the lack of referral based on these physical abnormalities remain unclear. At the time of their initial presentation at a mean age of 7 years, 8 months (range: 2 years, 11 months to 11 years, 9 months), $91 \%$ (29/32 patients with bone marrow biopsy reports) of patients already had histopathological evidence of bone marrow aplasia.

Compared with a cohort of healthy black South African children as published by Kimani-Murage et al., ${ }^{12}$ patients with FA were found to be significantly underweight (weight for age $z$-score $<-2$ ) and stunted (height for age $z$-score $<-2$ ) for age (Table 1).

Pigmentary anomalies, such as café au lait macules, hypopigmented streaks and macules, and hyperpigmented streaks and macules were detected on clinical evaluation. Although the pattern and distribution of the lesions differed between patients, $97 \%(34 / 35)$ of the affected individuals were noted to have at least one obvious pigmentary anomaly (Table 2).

Eye anomalies were commonly observed with all patients having at least one ocular anomaly. Epicanthic folds, upslanted palpebral fissure position, short palpebral fissures (shorter than $-2 \mathrm{SD}$ of the mean for age), and ptosis were the most frequent anomalies (Table 3).

The most notable ear anomalies in the study cohort were short pinna length for age $(<-2 \mathrm{SD}$ below the mean for age) and low set ears (Table 3 ).

Minor malformations of the radial and ulna rays of the upper limbs were frequently observed (Table 3 ). None of the 35 patients in the research cohort were found to have radial hypoplasia or aplasia (clinically or on X-ray evaluation (X-rays available in $24 / 35(69 \%))$. Lower-limb anomalies were infrequent $(<5 \%)$.

Renal system and gastrointestinal malformations, which occurred in $37 \%(12 / 31)$ and $8.5 \%(3 / 35)$ of the patients, respectively, were the most frequently noted major organ malformations. The renal anomalies included pelvic kidney $(6 / 31$ (19\%)), hypoplastic kidney (3/31 (9.7\%)), duplex collecting system (2/31 (6.5\%)), and ectopic kidney ((1/31) (3\%)). Gastrointestinal malformations included tracheoesophageal fistula, imperforate anus, and umbilical hernia. Malformations of the cardiovascular, central nervous system, genitalia, and vertebrae occurred infrequently $(<5 \%)$. Although the Tanner pubertal stage was documented in all the patients, the results are not reported as most patients were already on androgen therapy at the time of the examination which confounded the results.

\section{DISCUSSION}

Although FA is an extremely well-characterized genetic condition worldwide, in South African black patients it is usually only recognized late in the disease course at a stage when the symptoms of bone marrow aplasia are already evident. This suggests that despite the presence of growth disturbances and congenital malformations, the diagnosis of FA is not considered before the onset of bone marrow disease. Underrecognition and underdiagnosis of FA in South Africa is evidenced by a large discrepancy between the expected and observed number of FA cases referred to tertiary hematology/oncology centers. Unpublished data, based on gene frequency and population statistics, suggest that 14 out of 15 cases of FA do not reach tertiary care units and are probably not diagnosed. The reasons for the poor referral to tertiary care centers remain unclear, although the lack of a well-structured primary health-care system and pediatric, hematology, or clinical genetic expertise in rural and semi-urban settings may be contributing factors. The health-care system in South Africa is further overburdened by infectious diseases leading to a negative health-care transition.

Table 1 Frequency of underweight for age and stunting in FA and healthy South African children, stratified by age

\begin{tabular}{|c|c|c|c|c|c|c|c|c|}
\hline \multicolumn{3}{|c|}{ Weight for age $z$-score $<-2$} & \multicolumn{6}{|c|}{ Height for age $z$-score $<-2$} \\
\hline \multicolumn{3}{|c|}{ Age $<10$ years $^{a}$} & \multicolumn{3}{|c|}{ Age $<10$ years } & \multicolumn{3}{|c|}{ Age $>10$ years } \\
\hline FA & Healthy ${ }^{b}$ & $P$ value* & FA & Healthy & $P$ value* & FA & Healthy & $P$ value* \\
\hline
\end{tabular}

FA, Fanconi anemia.

Weight for age $z$-scores can only be calculated for children younger than the age of 10 years as the WHO-Anthro and Anthro-plus calculators do not allow weight for age $z$-scores to be calculated in children older than 10 years of age. ${ }^{b}$ Frequency values in healthy black South African children obtained from ref. $12 .{ }^{*} P$ values calculated with Fisher's exact test and deemed significant if $<0.05$.

Table 2 Pigmentary anomalies observed in black FA patients

1

Single visualized pigmentary anomaly

FA, Fanconi anemia. 
On the basis of the results of this study, certain characteristics have been found to be common components of the physical phenotype of FA in black South African patients. Some of these features may be useful in primary health-care settings in screening individuals for FA whereas others appear to require dysmorphology and clinical genetics experience to detect.

Individuals, homozygous for the founder mutation, were generally found to have low weight, height, and head circumference for age, and when compared to a cohort of healthy black South African children, were shown to be significantly underweight and stunted for age. Short stature is reported in up to $40 \%$ of other FA cohorts of heterogeneous genotype. ${ }^{2}$ Growth restriction should be a useful physical feature for FA screening as all children with short stature or microcephaly should be referred for a pediatric assessment and further investigations and consultation if shown to be pathological. ${ }^{13}$

All affected individuals were shown to have at least one ocular anomaly and most were found to have a minor ear malformation. The frequencies of eye and ear anomalies reported in the research cohort appear to be discrepant with those of the International Fanconi Anemia Registry cohort published by Auerbach et al., ${ }^{14}$ but in keeping with figures recently published by Tsilou et al. ${ }^{15}$ and Shimamura and Alter. ${ }^{16}$ Given the genetic heterogeneity of the patient cohorts and the variable research methodologies used in these three studies, it is not surprising that the frequency figures for eye and ear anomalies are so discrepant between the different groups (Table 3). Of note, while many patients in the black South African cohort were found to have short palpebral fissure length and short pinna length for age, when adjusted for head size in those who were microcephalic, these measurements fell within the normal range. The facial gestalt may thus not be easily recognizable in the growth-restricted child, particularly by health-care practitioners without dysmorphology experience.

Pigmentary anomalies (café au lait macules, hypo-, and hyperpigmented streaks and macules) were almost universally noted, with a frequency (97\%) that is significantly higher than has been reported internationally in genetically heterogeneous cohorts. ${ }^{14,17}$ The development of the lesions did not appear to be age- or sun exposure-dependent with most parents/guardians stating that the lesions had been present since birth. Hypopigmented lesions may be more easily discerned in individuals with darker skin tone. Although multiple pigmentary anomalies are not specific to FA, they should prompt further assessment with consideration of FA, particularly in the growth-restricted child.

Subtle anomalies of the radial and ulna ray of the upper limbs were noted in a high proportion of patients $(>70 \%)$, although these features may not be readily recognized by primary care practitioners or those not targeting their examination for the detection of features such as clinodactyly, short fifth digits, proximal insertion of the thumb, hypoplasia of the thumb, and increased mobility at the metacarpo-phalangeal joint of the thumb. Of particular note, none of the 35 patients in the research cohort were found to have radial hypoplasia or aplasia. This is often considered a central clinical characteristic of FA and reported in up to $7 \%$ of affected individuals of heterogeneous genotype. ${ }^{2}$ In the context of FA in black South African patients, radial hypoplasia cannot be used as a defining physical characteristic and may thus contribute to the under-ascertainment of affected individuals. The typically minor ulna and radial ray anomalies are unlikely to be of use to medical practitioners in rural and semi-urban settings owing to their difficulty to detect; however, they may be of use to pediatricians

Table 3 Nature and frequency of eye, ear, and hand anomalies in black FA patients and comparisons with other FA cohorts of heterogeneous genotype

\begin{tabular}{|c|c|c|c|c|c|}
\hline \multirow[b]{2}{*}{ Eye anomalies } & \multirow{2}{*}{$\begin{array}{l}\text { Specific anomaly } \\
\text { Upslanted palpebral fissure } \\
\text { position }\end{array}$} & \multirow{2}{*}{$\begin{array}{c}\text { Frequency in black } \\
\text { South African cohort } \\
28 / 35(80 \%)\end{array}$} & \multirow{2}{*}{$\begin{array}{c}\begin{array}{c}\geq 1 \text { Feature in an } \\
\text { individual }\end{array} \\
35 / 35(100 \%)\end{array}$} & \multicolumn{2}{|c|}{$\begin{array}{l}\text { Combined frequency of anomalies in FA } \\
\text { cohorts of heterogeneous genotype }\end{array}$} \\
\hline & & & & $21 / 22(95 \%)^{b}$ & $77 / 202(38.1 \%)^{c}$ \\
\hline & $\begin{array}{l}\text { Short palpebral fissure } \\
\text { length }\end{array}$ & $14 / 35(40 \%)$ & & & \\
\hline Ear anomalies & Short pinna length & $12 / 35(34.3 \%)$ & & $P=0.0001$ & \\
\hline \multirow[t]{3}{*}{ Hand anomalies } & Short 5th digit & $29 / 35(82.8 \%)$ & Ulna ray: 33/35 (94.3\%) & No data available & $35 \%^{d}$ \\
\hline & Clinodactyly & 23/35 (65.7\%) & & & \\
\hline & Proximally inserted thumb & $18 / 35(51.4 \%)$ & Radial ray: 26/35 (74.3\%) & $103 / 202(51 \%)^{c}$ & \\
\hline
\end{tabular}

FA, Fanconi anemia; SA, South African.

a'Compared with SA cohort using Fisher's exact test; $P$ value $<0.05$ significant. ${ }^{\text {bRef. } 15 . ~ ' R e f .14 . ~}{ }^{\text {dRef. }} 16$ - only percentages given, therefore unable to perform Fisher's exact test. 
and medical geneticists when considering a possible diagnosis of FA.

The frequency of renal anomalies in black South African FA patients (37\%) appears to be much higher than previously reported figures that suggest an incidence of renal malformations of $21 \%{ }^{2}$ The high incidence of renal anomalies in this cohort reinforces the need for a renal ultrasonographic evaluation in all black patients diagnosed with FA. The gastrointestinal malformations that were noted have all previously been reported in other FA cohorts. ${ }^{2,18}$

From a diagnostic perspective, recognition of the physical phenotype of black South African patients with FA remains a challenging task. The clinical evaluation of the 35 patients included in this study has shown that while growth and pigmentary abnormalities are significant physical features in most patients, other anomalies are subtle and may well be missed by those without experience in dysmorphology assessments. The reason for the underrecognition of the phenotype in black South African patients may in part be related to the subtle clinical features present in these patients, including the lack of radial aplasia, which differ from the classic features widely described.

A further factor hampering the diagnosis of FA may be the poor definition of the more severe FA phenotype. Patients with a severe presentation may die at an early age of congenital malformations or early onset bone marrow failure. A fetus that died in utero with multiple congenital abnormalities and bifid thumbs was found on molecular testing to be homozygous for the black South African founder mutation and was thus diagnosed post mortem with FA (unpublished data). This case suggests that a severe perinatal lethal form of the condition exists, but remains to be fully characterized.

The link between FA and the VACTERL (vertebral, ano-rectal, cardiac, tracheoesophageal, renal, and limb malformations) association has been evaluated, with emphasis being placed on the overlap in physical phenotype between the two conditions. Faivre et al. ${ }^{19}$ suggest that upto $5 \%$ of patients with FA fulfill the criteria for VACTERL association and that FA testing should be undertaken in individuals with VACTERL who have radial ray anomalies or other features such as growth retardation, pigmentary anomalies, microcephaly, or dysmorphism. In the South African context, unpublished data suggest that the founder FANCG mutation is uncommon in cases identified with VACTERL association. Reasons for this may include the low incidence of radial hypoplasia and aplasia in affected black individuals as well as the low frequency of congenital cardiac and vertebral malformations.

At this stage, it remains difficult to answer the question of whether black individuals homozygous for the FANCG founder mutation have a physical phenotype distinctly recognizable from other individuals with FA. Given the heterogeneity of mutations implicated in the causation of FA and the diverse spectrum of clinical manifestations, researchers have previously attempted to establish genotype-phenotype correlations. Many of these research efforts have focused mainly on the effect of various mutations in specific genes on hematological outcome and in some cases on the extent of physical malformations. ${ }^{17,20,21}$ Researchers have differentially found that individuals with either FANCG or FANCC mutations are at greater risk of severe cytopenia, whereas those with mutations in either FANCA or FANCG tend to have more somatic anomalies including skin and skeletal anomalies. ${ }^{17,22}$ Genes appear to have been used as a proxy for specific mutations in most studies presumably because of the marked heterogeneity of FA with many mutations described within each causative gene and the difficulty in recruiting a patient cohort who are genetically homogeneous. Neveling et al. (2009), ${ }^{23}$ in a review article, focused on the genotype-phenotype correlations in FA and pointed out the futility in attempting these correlations using individual genes/complementation groups rather than specific mutations.

Few mutation-specific genotype-phenotype correlation studies have been performed and in most cases the study cohorts include those homozygous and heterozygous for the mutation in question. Table 4 documents a comparison of the frequency of certain abnormalities in individuals with specific FANCA and FANCC mutations to the frequency in this research cohort. Of note, these studies group some anomalies anatomically

Table 4 Frequency of somatic abnormalities in FA patients with common FANCA and FANCC mutations and comparison to patients with founder FANCG mutation

\begin{tabular}{|c|c|c|c|c|}
\hline \multirow[b]{2}{*}{ Abnormality } & \multicolumn{4}{|c|}{ Mutation } \\
\hline & FANCA del E12-31 & $\begin{array}{l}\text { FANCC IVS4 + 4A }>\text { T } \\
\text { (non-Japanese) }^{\mathrm{a}}\end{array}$ & $\begin{array}{l}\text { FANCC IVS4 + 4A }>\text { T } \\
\text { (Japanese) }^{\mathrm{b}}\end{array}$ & FANCG 637_643delTACCGCC \\
\hline Pigmentary anomaly & $38 / 44(86 \%)$ & - & $19 / 29(65 \%)$ & $34 / 35(97 \%)$ \\
\hline Head anomalies ${ }^{c}$ & $34 / 44(77 \%)$ & - & $8 / 29(27 \%)$ & $35 / 35(100 \%)$ \\
\hline Radial ray anomalies & $32 / 44(72 \%)$ & $4 / 5(80 \%)$ & $15 / 29(52 \%)$ & $26 / 35(74 \%)$ \\
\hline Kidney malformations & - & $4 / 5(80 \%)$ & $2 / 29(7 \%)$ & $12 / 31(37 \%)$ \\
\hline CNS anomalies & - & $4 / 5(80 \%)$ & - & $0 / 35$ \\
\hline
\end{tabular}

CNS, central nervous system; FA, Fanconi anemia; GIT, gastrointestinal tract.

Frequency of anomalies in FANCG cohort compared with frequency of anomalies in FANCA and two FANCC cohorts using Fisher's exact test. Significant $P$ value $<0.05$

highlighted in bold. ${ }^{a}$ Ref. 19. ${ }^{\text {b Ref.24. }}$ 'Head anomalies include microcephaly, micrognathia, a triangular face, and eye and ear anomalies (as described by ref.19). 
rather than describing specific features in detail. The FANCG cohort appear to have a significantly higher incidence of pigmentary and renal anomalies than the Japanese cohort and a higher incidence of "head" anomalies (which included microcephaly, eye anomalies, and ear anomalies) than both the FANCA and Japanese FANCC cohorts. It can be noted that Japanese and non-Japanese individuals with the same FANCC mutation have been shown to have variable phenotypes, with Japanese individuals displaying a milder phenotype with fewer major anomalies. This suggests that the clinical phenotype of FA is also influenced by ethnic background ${ }^{24}$ and other genetic factors. There are, as yet, no studies documenting the physical phenotype of the $20 \%$ of black FA patients who do not have FANCG mutations. Examination of this small group of patients and a comparative analysis to the phenotype in individuals homozygous for the founder mutation would possibly provide further insights into FA in black South African patients.

Although this research represents the largest group of black FA patients with a single underlying genetic mutation in South Africa and is the first mutation-based genotype-physical phenotype correlation study in black South African patients, various limitations need to be addressed. A group of healthy black South African patients was not used as a control in this phase of the research. Rather, the phenotype of the black patients was compared with previously reported data from international FA cohorts and growth data from a healthy black South African cohort published in 2010. The second phase of this project is underway to evaluate healthy black South African children and compare their phenotype with that of the FA patients. This phase of the research aims to determine whether the described physical phenotype is unique to patients homozygous for the founder mutation or if some of the features are related to the common ethnicity of the affected individuals. Furthermore, as no specific growth and physical parameter charts have been derived for black South African or African patients, all reference materials used are those that are currently used in standard pediatric and clinical genetic practice in South Africa which is derived from a predominantly Caucasian population group.

Second, only FA patients attending tertiary care facilities were recruited to participate. We may thus have ascertained more severely affected individuals requiring therapeutic intervention and may have missed mildly affected individuals with minor anomalies and slow progression to bone marrow disease, as well as the most severely affected individuals who die before referral to a tertiary centre. However, given the underdeveloped health-care system in South Africa and the poor accessibility of patients attending rural and semi-urban primary health-care clinics to tertiary centers, this limitation cannot be overcome at the present time.

\section{CONCLUSION}

FA in the black population in South Africa is caused by a founder mutation in the FANCG gene in the majority of cases. Individuals homozygous for this mutation have been shown to have significant growth restriction and a high incidence of pigmentary and renal anomalies compared with other FA cohorts. The physical features in black patients appear to be subtle and may require dysmorphology experience to detect. These findings may in part explain the poor recognition of the FA phenotype at primary and secondary care level. Education of health-care practitioners in the recognition of the phenotype remains challenging given the lack of very distinct features in affected individuals as well as the vast infectious and nongenetic health burdens in the public health-care sector which take priority in terms of care.

In South Africa, the number of medical geneticists is currently at a critically low level (13 trained medical/clinical geneticists; SA population $>50$ million) and so the onus is on nurses and general practitioners at primary care level to refer suspected patients for an assessment. To improve the diagnostic pick up rate of FA, we would suggest that all individuals with growth restriction, particularly in association with skin pigmentary anomalies, be referred for a pediatric assessment and then a medical genetics consultation should it be required. Even though most of these children will not be shown to have FA, a pediatric assessment on the basis of short stature or microcephaly alone is justified.

Additional investigation into the spectrum of disease severity associated with this founder mutation is still required. Research into the hematological phenotype of this cohort has been performed and will be reported on separately.

\section{ACKNOWLEDGMENTS}

The authors thank Prof. Elena Libhaber for her assistance with statistical analysis; Drs. Tshvase and Surtee for the interpretation of X-ray and ultrasound findings; clinical, counseling, laboratory, and support staff in the Department of Human Genetics (National Health Laboratory Service and The University of the Witwatersrand); clinical and support staff at Chris Hani Baragwanath Hospital, Charlotte Maxeke Johannesburg Academic Hospital, Universitas Hospital, and Polokwane/Mankweng Hospital Complex; and the patients and their families who agreed to participate in this research.

\section{DISCLOSURE}

The authors declare no conflict of interest.

\section{REFERENCES}

1. Meetei AR, Levitus $M, X u e Y$, et al. X-linked inheritance of Fanconi anaemia complementation group B. Nature Genet 2004;36:1219-1224.

2. Alter B, Kupfer G. Fanconi Anaemia. Gene Reviews. Last updated February 2011. http://www.ncbi.nlm.nib.gov/books/NBK1401 Accessed 4 April 2011.

3. De Kerviler E, Guermazi A, Zagdanski AM, Gluckman E, Frija J. The clinical and radiological features of Fanconi's anaemia. Clin Radiol 2000;55: 340-345.

4. Giri N, Batista DL, Alter BP, Stratakis CA. Endocrine abnormalities in patients with Fanconi anemia. J Clin Endocrinol Metab 2007;92:2624-2631.

5. Tischkowitz MD, Hodgson SV. Fanconi anaemia. J Med Genet 2003;40:1-10.

6. Rosenberg PS, Greene MH, Alter BP. Cancer incidence in persons with Fanconi anemia. Blood 2003;101:822-826.

7. de Winter JP, Joenje $\mathrm{H}$. The genetic and molecular basis of Fanconi anemia Mutat Res 2009;668:11-19.

8. Auerbach AD. Fanconi anemia and its diagnosis. Mutat Res 2009;668:4-10.

9. Morgan NV, Essop F, Demuth I, et al. A common Fanconi anemia mutation in black populations of sub-Saharan Africa. Blood 2005;105:3542-3544. 
10. Macdougall LG, Greeff MC, Rosendorff J, Bernstein R. Fanconi anemia in black African children. Am J Med Genet 1990;36:408-413.

11. Hall JG, Allanson J, Gripp K, et al. Handbook of Physical Measurements, 2nd edn. Oxford University Press: New York.

12. Kimani-Murage EW, Kahn K, Pettifor JM, et al. The prevalence of stunting, overweight and obesity, and metabolic disease risk in rural South African children. BMC Public Health 2010:10:158.

13. Westwood T, Kibel M, Saloojee H. Child Health for All, 4th edn. Oxford University Press: Cape Town, Southern Africa.

14. Auerbach AD, Rogatko A, Schroeder-Kurth TM. International Fanconi Anemia Registry: relation of clinical symptoms to diepoxybutane sensitivity. Blood 1989;73:391-396.

15. Tsilou ET, Giri N, Weinstein S, Mueller C, Savage SA, Alter BP. Ocular and orbital manifestations of the inherited bone marrow failure syndromes: Fanconi anemia and dyskeratosis congenita. Ophthalmology 2010;117:615-622.

16. Shimamura A, Alter BP. Pathophysiology and management of inherited bone marrow failure syndromes. Blood Rev 2010;24:101-122.

17. Faivre L, Guardiola P, Lewis C, et al. Association of complementation group and mutation type with clinical outcome in fanconi anemia. European Fanconi Anemia Research Group. Blood 2000;96:4064-4070.
18. Giampietro PF, Adler-Brecher B, Verlander PC, Pavlakis SG, Davis JG, Auerbach AD. The need for more accurate and timely diagnosis in Fanconi anemia: a report from the International Fanconi Anemia Registry. Pediatrics 1993;91:1116-1120.

19. Faivre L, Portnoï MF, Pals G, et al. Should chromosome breakage studies be performed in patients with VACTERL association? Am J Med Genet A 2005;137:55-58

20. Gillio AP, Verlander PC, Batish SD, Giampietro PF, Auerbach AD. Phenotypic consequences of mutations in the Fanconi anemia FAC gene: an International Fanconi Anemia Registry study. Blood 1997;90: 105-110.

21. Rosenberg PS, Huang Y, Alter BP. Individualized risks of first adverse events in patients with Fanconi anemia. Blood 2004;104:350-355.

22. Kutler DI, Singh B, Satagopan J, et al. A 20-year perspective on the International Fanconi Anemia Registry (IFAR). Blood 2003;101:1249-1256.

23. Neveling K, Endt D, Hoehn H, Schindler D. Genotype-phenotype correlations in Fanconi anemia. Mutat Res 2009;668:73-91.

24. Futaki M, Yamashita T, Yagasaki H, et al. The IVS4 + 4 A to T mutation of the fanconi anemia gene FANCC is not associated with a severe phenotype in Japanese patients. Blood 2000;95:1493-1498. 